\title{
Enhanced response of non-Hermitian photonic systems near exceptional points
}

\author{
Satoshi Sunada \\ Faculty of Mechanical Engineering, Institute of Science and Engineering, \\ Kanazawa University, Kakuma-machi Kanazawa, Ishikawa 920-1192, Japan
}

(Dated: June 8, 2021)

\begin{abstract}
This paper theoretically and numerically studies the response characteristics of non-Hermitian resonant photonic systems operating near an exceptional point (EP), where two resonant eigenmodes coalesce. It is shown that a system near an EP can exhibit a non-Lorentzian frequency response, whose line shape and intensity strongly depend on the modal decay rate and coupling parameters for the input waves, unlike a normal Lorentzian response around a single resonance. In particular, it is shown that the peak intensity of the frequency response is inversely proportional to the fourth power of the modal decay rate and can be significantly enhanced with the aid of optical gain. The theoretical results are numerically verified by a full wave simulation of a microring cavity with gain. In addition, the effects of the nonlinear gain saturation and spontaneous emission are discussed. The response enhancement and its parametric dependence may be useful for designing and controlling the excitation of eigenmodes by external fields.
\end{abstract}

\section{INTRODUCTION}

In a quantum system interacting with the surrounding environment, the quantum property is described by a non-Hermitian Hamiltonian formalism [1]. The eigenvalues of the non-Hermitian Hamiltonian are generally complex, and the eigenmodes do not form an orthogonal basis, unlike those of isolated (Hermitian) systems. Such non-Hermitian properties also appear in classical wave systems such as acoustic or electromagnetic waves in open cavities.

A remarkable feature of non-Hermitian systems is the nonorthogonality of the eigenmodes. When a nonHermitian system is driven by noise, the nonorthogonality can lead to excess system noise whose amplitude can greatly exceed the level expected in Hermitian systems. To date, many theoretical and experimental works in different contexts, including Bose-Einstein condensates, lasers, fluid dynamics, and pattern formation, have revealed that excess noise is a common feature of nonnormal (and non-Hermitian) physical systems driven by noise and manifests itself in certain forms [2 9 ].

In the context of laser physics, excess spontaneous emission noise in laser cavities has been well-studied [1016]. The excess noise has been observed as the broadening of the laser linewidth [17-20] and a low-frequency intensity fluctuation [21], and it has been characterized by the Petermann factor (PF), a measure of the nonorthogonality of the eigenmodes. Importantly, the PF does not characterize the enhancement in the spontaneous emission itself but rather the enhanced coupling of the spontaneous emission to an eigenmode [14, 15]. This implies that the PF can be generalized as a factor characterizing the enhanced response of an eigenmode to inputs, and the excess spontaneous emission is one aspect of the enhanced response. Actually, a system with a large PF can exhibit an excess excitation response for external injection in an amplifier configuration [11 13].

It is known that the modal nonorthogonality is maximized and the PF diverges at a degeneracy point called an exceptional point (EP), where both the eigenvalues and corresponding eigenmodes coalesce [16, 22 24]. Recently, EPs have become experimentally accessible in a variety of photonic systems [25-28] and have attracted much attention. In addition to the PF divergence, a number of unique properties related to EPs have been found and demonstrated in the past few years, such as asymmetric mode switching [29], the reversal of the pump dependence of a laser 30 and the effect of loss 31], nonreciprocal transmission [32, 33], and unidirectional invisibility 34, 35]. Moreover, EPs have been used to enhance the sensitivity of microcavity sensors [36 40].

In this paper, the response characteristics of nonHermitian resonant systems (optical cavities) near an EP are theoretically and numerically studied. A linear response analysis near an EP reveals that regardless of the $\mathrm{PF}$, the actual response intensity is limited to a finite value. Instead, a variety of cavity responses to inputs are exhibited near an EP, mainly depending on the modal decay rates and coupling parameters for the input waves. In particular, when an optical cavity with gain operates at an EP, the response intensity can be excessively enhanced with the aid of the gain. The condition for the enhancement is derived and discussed. These theoretical results are numerically verified by a dynamical model describing the interaction between the light field and a two-level gain medium. It is also discussed that the nonlinear gain saturation and spontaneous emission limit the enhancement in the response intensity and quality.

The rest of this paper is organized as follows. In Sec. III the effect of the nonorthogonality of the eigenmodes on the system response is briefly introduced. Then, a general expression of the system response at an EP is provided in a simple $2 \times 2$ matrix form, and the frequency responses are analyzed. In Sec. III, the theoretical results are numerically verified in a full wave simulation of a microring cavity operating near an EP. Finally, a summary is provided in Sec. IV] 


\section{LINEAR RESPONSES OF NON-HERMITIAN SYSTEMS NEAR AN EP}

\section{A. Model and Petermann factor}

We consider an optical cavity system driven by an input field and analyze the response characteristics in the form of a coupled mode theory. First, suppose that an optical cavity possesses $n$ modes coupled with each other and that it is described by an $n \times n$ non-Hermitian (effective Hamiltonian) matrix $\hat{H}$, which represents the resonances and modal coupling. The non-Hermiticity of $\hat{H}$ arises from the radiation loss, absorption loss, and gain inside the cavity. Then, we also suppose that the slowly varying envelope of the intracavity optical field is characterized by an $n$-dimensional state vector $\psi \in \mathbb{C}^{n}$, and it is excited by an input field, denoted by $\boldsymbol{f} \in \mathbb{C}^{n}$. The time evolution of $\boldsymbol{\psi}$ driven by $\boldsymbol{f}$ is generally given by

$$
i \frac{d \boldsymbol{\psi}}{d t}=\hat{H} \boldsymbol{\psi}+\boldsymbol{f} .
$$

This coupled mode equation can be obtained from the Maxwell equations by assuming that the optical field varies slowly in time with respect to a reference frequency and it is expanded by appropriate basis functions. In this study, we are interested in how the state vector $\boldsymbol{\psi}$ responds to an input $\boldsymbol{f}$ because typical cavity properties such as reflection or transmission can be characterized by the response $\boldsymbol{\psi}$.

A common way to analyze Eq. (11) is an expansion by the eigenmodes of $\hat{H}$. Although the eigenmodes of a non-Hermitian matrix are generally not orthogonal, this drawback is covered by the biorthogonality between the left and right eigenmodes. Here, suppose that $\boldsymbol{u}_{j}$ and $\boldsymbol{v}_{j}$ are the right and left eigenmode vectors of $\hat{H}$, respectively, where $j$ is a mode number $(j \in\{1, \cdots, n\})$. $\boldsymbol{u}_{j}$ and $\boldsymbol{v}_{j}$ are defined as $\hat{H} \boldsymbol{u}_{j}=\Omega_{j} \boldsymbol{u}_{j}$ and $\boldsymbol{v}_{j}^{\dagger} \hat{H}=\Omega_{j} \boldsymbol{v}_{j}^{\dagger}$ with the eigenvalue $\Omega_{j} \in \mathbb{C}$, where $\dagger$ denotes the Hermitian conjugate, and $\boldsymbol{v}_{i}^{\dagger} \cdot \boldsymbol{u}_{j}=0(i \neq j)$. The eigenvalue $\Omega_{j}=\omega_{j}-i \gamma_{j}$ represents a complex-valued eigenfrequency of mode $j$ in the cavity; $\omega_{j} \in \mathbb{R}$ represents the resonant frequency, whereas $\gamma_{j} \in \mathbb{R}$ represents the decay (growth) rate if it has a positive (negative) value. In this paper, we consider only $\gamma_{j}>0$ for all $j$, i.e., all decaying modes.

By expanding $\boldsymbol{\psi}$ by the right eigenmodes as $\boldsymbol{\psi}=$ $\sum_{j} a_{j}(t) \boldsymbol{u}_{j}$ and using the biorthogonal relation in Eq. (11), we obtain the mode equations,

$$
i \frac{d a_{j}}{d t}=\Omega_{j} a_{j}+f_{j}
$$

and the solution after a long time, $a_{j}(t)=$ $-i \int^{t} e^{-i \Omega_{j}(t-\tau)} f_{j}(\tau) d \tau$, where $f_{j}=\boldsymbol{v}_{j}^{\dagger} \cdot \boldsymbol{f} /\left(\boldsymbol{v}_{j}^{\dagger} \cdot \boldsymbol{u}_{j}\right)$. Importantly, the amplitude $a_{j}$ is determined by $f_{j}$, and the magnitude $\left|f_{j}\right|$ can be expressed as $\sqrt{K_{j}} \mid \boldsymbol{v}_{j}^{\dagger}$. $\boldsymbol{f} \mid /\left(\left\|\boldsymbol{u}_{j}\right\|\left\|\boldsymbol{v}_{j}\right\|\right)$, where $K_{j}=\left(\left\|\boldsymbol{u}_{j}\right\|^{2}\left\|\boldsymbol{v}_{j}\right\|^{2}\right) /\left|\boldsymbol{v}_{j}^{\dagger} \cdot \boldsymbol{u}_{j}\right|^{2}$, and $\|\cdot\|$ denotes the usual Euclidean vector norm. $K_{j}$ characterizes the coupling to mode $j$, and it is associated with the condition number of the eigenvalue $\Omega_{j}$, i.e., the sensitivity to perturbations [2, 41] and the PF in the context of laser physics [20, 23]. $K_{j} \geq 1$ is always satisfied according to the Cauchy-Bunyakovsky-Schwarz inequality. In particular, $K_{j}>1$ when $\hat{H}$ is nonnormal $\left(\hat{H} \hat{H}^{\dagger} \neq \hat{H}^{\dagger} \hat{H}\right)$ [42]. Interestingly, $K_{j}$ diverges just at an EP, where $\boldsymbol{u}_{j}$ completely overlaps another eigenvector, e.g., $\boldsymbol{u}_{j^{\prime}}\left(j^{\prime} \neq j\right)$, because of the self-orthogonality $\boldsymbol{v}_{j}^{\dagger} \cdot \boldsymbol{u}_{j}=\boldsymbol{v}_{j}^{\dagger} \cdot \boldsymbol{u}_{j^{\prime}}=0$ [16]. However, $K_{j}$ is no longer valid at the EP because the eigenmode expansion breaks at the point. In other words, any $n$-dimensional state vector cannot be represented by the coalescing eigenmode basis at the EP. The basis of the expansion can be completed by introducing additional vectors, i.e., the associated vectors defined by the Jordan chain relations [43, 44].

\section{B. Frequency response at an EP}

As a starting point for deriving the cavity responses at an EP, we consider them in the frequency domain. By Fourier-transforming Eq. (1) with respect to the time $t$, we obtain

$$
\tilde{\boldsymbol{\psi}}(\omega)=\hat{M}(\omega) \tilde{\boldsymbol{f}}(\omega),
$$

where $\tilde{\boldsymbol{\psi}}(\omega)$ and $\tilde{\boldsymbol{f}}(\omega)$ denote the Fourier transforms of $\boldsymbol{\psi}(t)$ and $\boldsymbol{f}(t)$, respectively. $\hat{M}(\omega)=(\omega \hat{I}-\hat{H})^{-1}$ is the resolvent of $\hat{H}$ and characterizes the response to an input wave with a frequency of $\omega . \hat{I}$ is an identity matrix.

Then, we consider an optical cavity operating near a (second-order) EP, where only two eigenmodes of $\hat{H}$ coalesce. When the frequency $\omega$ is close to the resonant frequencies of the two eigenmodes and the influence of the other $n-2$ modes is sufficiently weak, the $n$-dimensional matrix problem can be essentially reduced to a twodimensional matrix problem near the EP. By describing an effective $2 \times 2$ Hamiltonian matrix at an EP as $\hat{H}_{0}$ and using an expansion method based on the Jordan chain relation [45], the resolvent $\hat{M}_{E P}$ at the EP is given by

$$
\begin{array}{r}
\hat{M}_{E P}(\omega)=R_{0}(\omega) \hat{I}+R_{0}^{2}(\omega)\left(\hat{H}_{0}-\Omega_{0} \hat{I}\right) \\
=\left(\begin{array}{rr}
R_{0}(\omega)+c_{11} R_{0}^{2}(\omega), & c_{12} R_{0}^{2}(\omega) \\
c_{21} R_{0}^{2}(\omega), & R_{0}(\omega)+c_{22} R_{0}^{2}(\omega)
\end{array}\right),
\end{array}
$$

where $R_{0}(\omega)=\left(\omega-\Omega_{0}\right)^{-1}$, and $\Omega_{0}$ is an eigenvalue of $\hat{H}_{0}$ at the EP. Further, $c_{i j}$ is the $i j$ component of the matrix $\left(\hat{H}_{0}-\Omega_{0} \hat{I}\right)$. The derivation of Eq. (4) is shown in Appendix $\mathrm{A}$

A remarkable feature of the resolvent $\hat{M}_{E P}$ is the presence of the second-order pole $R_{0}^{2}$, which appears only near the EP. From Eqs. (3) and (4), the $i$ component of the field vector, $\tilde{\psi}_{i}(\omega)$, driven by $\tilde{\boldsymbol{f}}(\omega)=\left(\tilde{f}_{1}, \tilde{f}_{2}\right)^{T}$, is $\tilde{f}_{i} R_{0}+\delta_{i} R_{0}^{2}$, where $\delta_{i}=\sum_{j} c_{i j} \tilde{f}_{j}$. Interesting behav- 
ior can be observed for $\gamma_{0} \sim\left|\delta_{i} / \tilde{f}_{i}\right|$ or $\gamma_{0} \ll\left|\delta_{i} / \tilde{f}_{i}\right|$, where $\gamma_{0}=-\operatorname{Im} \Omega_{0}$. In the former case, the interference of the two terms can lead to asymmetric response behavior with respect to the resonant frequency, $\omega_{0}=\operatorname{Re} \Omega_{0}$, or suppression of the response amplitude, such as Fano-Feshbach resonances [46, 47]. On the other hand, in the latter case $\left(\gamma_{0} \ll\left|\delta_{i} / \tilde{f}_{i}\right|\right), \tilde{\psi}_{i}$ can be approximated as $\delta_{i} R_{0}^{2}$ near the resonant frequency $\omega_{0}$; thus, the intensity $\left|\tilde{\psi}_{i}\right|^{2}$ has a squared Lorentzian shape, i.e., $\left|\delta_{i} R_{0}^{2}\right|^{2}=\left|\delta_{i}\right|^{2} /\left[\gamma_{0}^{2}+\left(\omega-\omega_{0}\right)^{2}\right]^{2}$, with a peak intensity proportional to $\gamma_{0}^{-4}$, whereas the peak intensity of a standard Lorentzian shape is proportional to $\gamma_{0}^{-2}$. Although $\gamma_{0}$ is generally dependent on $c_{i}$ and the input wave couplings in passive cavities, it can be changed by optical gain in active cavities. Therefore, when $\gamma_{0}$ is reduced by the gain, the response intensity near the resonance can be greater than the standard Lorentzian-type responses in cavities that do not operate at an EP.

It has been reported that a similar enhancement appears for spontaneous emission [45, 48, 49]. The above discussion suggests that the enhanced spontaneous emission is one aspect of the enhanced cavity responses; a cavity operating at an EP can respond strongly to other inputs.

\section{Example of enhanced response}

As an example of an optical cavity that can operate at an EP, we choose a microring cavity with non-Hermitian backscattering [50 52] and analyze the cavity response to an incident wave with a frequency of $\omega$. The wave is coupled to the cavity via a waveguide [see Fig. 1. In the cavity, the wave can propagate in the clockwise (CW) or counterclockwise (CCW) direction along the ring waveguide. The time evolution of the intracavity field in the $\mathrm{CW}$ and $\mathrm{CCW}$ traveling wave basis is given by

$i \frac{d}{d t}\left(\begin{array}{c}a_{1} \\ a_{2}\end{array}\right)=\left(\begin{array}{cc}\Omega_{0} & -\epsilon / 2 \\ -p / 2, & \Omega_{0}\end{array}\right)\left(\begin{array}{c}a_{1} \\ a_{2}\end{array}\right)+\left(\begin{array}{c}\kappa_{1} \\ \kappa_{2}\end{array}\right) e^{-i \omega t},(5)$

where $a_{1}$ and $a_{2}$ are the amplitudes of the CCW and CW waves, respectively. $\kappa_{1}$ and $\kappa_{2}$ represent the coupling of the incident wave to the CCW and $\mathrm{CW}$ waves, respectively. $\Omega_{0}=\omega_{0}-i \gamma_{0}$ is the eigenfrequency of the ring cavity modes ( $\mathrm{CCW}$ and $\mathrm{CW}$ modes) when there are no coupling terms, i.e., $\epsilon=p=0 . \epsilon(p)$ represents the backscattering coupling from the $\mathrm{CW}$ (CCW) wave to the $\mathrm{CCW}(\mathrm{CW})$ wave. In general, the magnitudes and phases of $\epsilon$ and $p$ can be controlled by placing nanoscatterers near the cavity [50], making the cavity geometry asymmetric [51], or introducing modulations in the refractive index and dissipation inside a cavity [35, 52].

Backscattering coupling typically causes the splitting of the degenerate eigenvalues of the $\mathrm{CW}$ and $\mathrm{CCW}$ modes. However, when the backscattering couplings are highly asymmetric, i.e., $\epsilon \neq 0$ and $p=0$ or $\epsilon=0$ and $p \neq 0$, the split eigenvalues coalesce into a single value
51]. (In this cavity, the EPs are distributed along the line $p=0$ or $\epsilon=0$ except for the origin, $\epsilon=p=0$, in the $\epsilon-p$ parametric plane.) The coalesced eigenvalue is $\Omega_{0}$ at an EP, and the corresponding eigenmode is the CCW (CW) mode when $\epsilon \neq 0$ and $p=0(\epsilon=0$ and $p \neq 0)$. This non-Hermitian degeneracy is different from the degeneracy in ring cavities without backscattering, i.e., $\epsilon=p=0$, where there are two linearly independent eigenmodes (the CW and CCW modes) with the same eigenfrequency $\Omega_{0}$. The normal degeneracy point has been referred to as the diabolic point (DP).

At an $\mathrm{EP}(\epsilon \neq 0$ and $p=0)$, the $\mathrm{CW}$ and $\mathrm{CCW}$ wave amplitudes in the frequency domain are represented by

$$
\left(\begin{array}{c}
\tilde{a}_{1}(\omega) \\
\tilde{a}_{2}(\omega)
\end{array}\right)=\frac{1}{\omega-\Omega_{0}}\left(\begin{array}{cc}
1, & -\frac{\epsilon}{2\left(\omega-\Omega_{0}\right)} \\
0, & 1
\end{array}\right)\left(\begin{array}{l}
\kappa_{1} \\
\kappa_{2}
\end{array}\right) .
$$

The CCW wave amplitude, $\tilde{a}_{1}$, is affected by the backscattering $\epsilon$, decay rate $\gamma_{0}$, and coupling terms $\kappa_{1}$ and $\kappa_{2}$. When a wave with a frequency of $\omega \approx \omega_{0}$ is incident upon the cavity in the $\mathrm{CW}$ direction, i.e., $\kappa_{1}=0$ and $\kappa_{2} \neq 0$, the two-mode intensities near the resonance $\omega \approx \omega_{0}$ are given by $\left|\tilde{a}_{1}\left(\omega_{0}\right)\right|^{2} \approx\left|\epsilon \kappa_{2}\right|^{2} /\left(4 \gamma_{0}^{4}\right)$ and $\left|\tilde{a}_{2}\left(\omega_{0}\right)\right|^{2} \approx\left|\kappa_{2}\right|^{2} \gamma_{0}^{-2}$. Thus, $\left|\tilde{a}_{1}\left(\omega_{0}\right)\right|^{2} \gg\left|\tilde{a}_{2}\left(\omega_{0}\right)\right|^{2}$ for $\gamma_{0} \ll|\epsilon| / 2\left(=\gamma_{s}\right)$; that is, the CCW wave is strongly excited by the incident wave in the CW direction. This counterintuitive response does not arise in a ring cavity without backscattering $(\epsilon=p=0)$ or in cavities with isolated resonances. Numerical verification is presented in Sec. [III]

\section{Transient growth and excess noise}

Because the cavity responses are characterized by Eqs. (3) and (4), cavities operating near EPs can respond sensitively to not only monochromatic inputs but also other inputs [see Figs. 2(a) and (b) for examples of the responses to pulsed and random inputs]. From Eq. (3), we note that in the presence of internal noise such as spontaneous emission, the cavities can also be sensitive to the noise. This is excess noise in the cavity and limits the response quality.

When we consider the response properties in the time domain, we note that the impulse response corresponding to $R_{0}^{2}$ in Eq. (44) is given by $t \exp \left(-i \Omega_{0} t\right)$, whose amplitude grows transiently on a short time scale before decaying. As seen in Figs. 2(a) and (b), the transient growth is sustained by sequential pulses or noise stimuli. Excess noise can be characterized as the lasting transient growth due to internal noise. According to nonnormal operator theory 53], transient growth can dominate the dynamics of the intensity $\|\boldsymbol{\psi}\|^{2}$ if at least one eigenvalue of $i\left(\hat{H}^{\dagger}-\hat{H}\right)$ (the non-Hermitian part of $\hat{H}$ ) is positive. In the $2 \times 2$ matrix model at an EP, the condition is given 


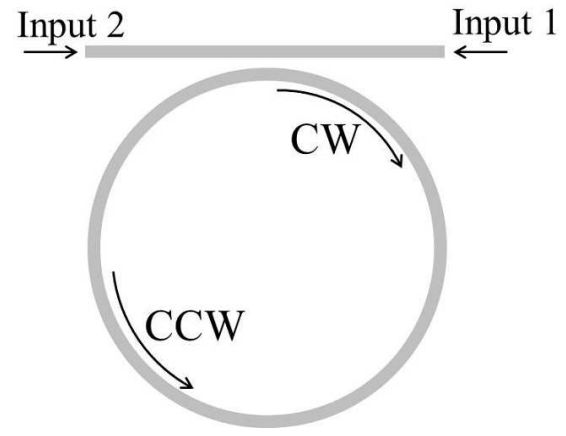

FIG. 1: Microring cavity coupled to a straight waveguide. The input waves from the left and right ports in the straight waveguide couple to the $\mathrm{CW}$ and $\mathrm{CCW}$ modes in the microring, respectively. The two modes are also coupled to each other by backscattering.

by

$$
\gamma_{0}<\gamma_{n}=\frac{1}{2} \sqrt{-\left(c_{11}+c_{22}^{*}\right)^{2}+\left|c_{21}^{*}-c_{12}\right|^{2}} .
$$

In a microring cavity at the $\mathrm{EP}(\epsilon \neq 0$ and $p=0)$, the condition is $\gamma_{0}<\gamma_{n}=|\epsilon| / 4$. Figure 2(c) shows the appearance of excess noise in a microring cavity, where the time-averaged response intensity to Gaussian noise at an EP $(\epsilon \neq 0$ and $p=0)$, represented by $I_{e p}$, is compared to that of the response intensity at a DP $(\epsilon=p=0)$, $I_{d p}$. When $\gamma_{0} \ll \gamma_{n}, I_{e p}$ is $\gamma_{n}^{2} / \gamma_{0}^{2}$ times greater than $I_{d p}$.

\section{FULL WAVE SIMULATIONS}

\section{A. Dynamical model}

In this section, we numerically check the validity of the response enhancement presented in the previous section. This numerical verification is important because the linear response model is based on a simple $(2 \times 2$ matrix) model, i.e., a two-mode approximation near an $\mathrm{EP}$; however, in realistic systems, multiple modes may be involved. Moreover, to reduce the decay rate $\gamma_{0}$ and achieve an excess enhancement in the response intensity, loss compensation by optical gain is needed. However, amplification by the gain is inevitably accompanied by spontaneous emission noise. In addition, an actual gain material is nonlinear and saturates the amplified light intensity. Therefore, the numerical simulations are conducted using a dynamical model incorporating these effects. In this paper, we use a model describing the dynamics of the slowly varying envelope $E$ of the electric field inside a cavity, the polarization field $\rho$, and the population inversion component $W$ in a two-level gain
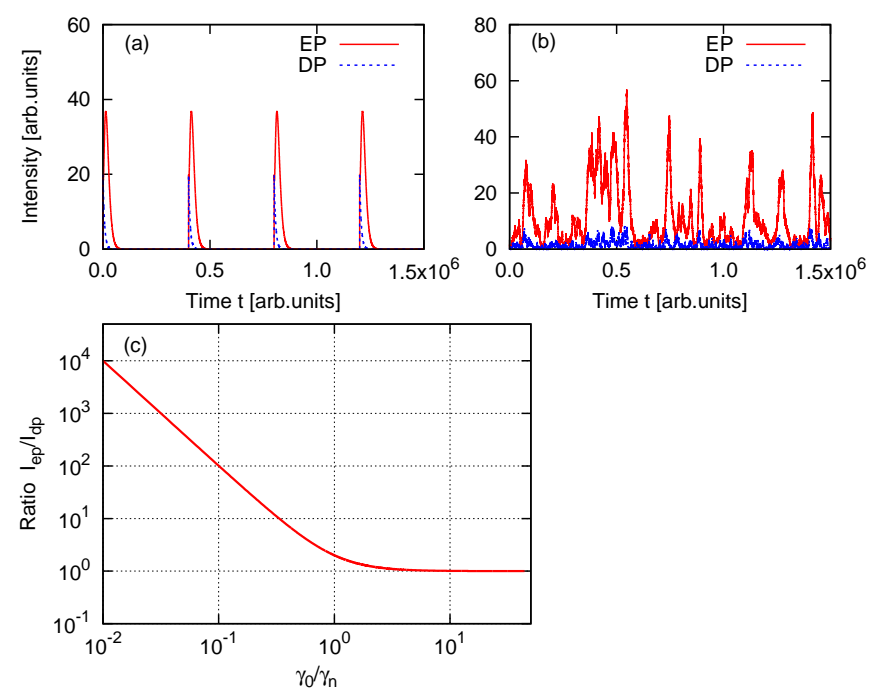

FIG. 2: (a), (b) Dynamics of the intensity $\|\boldsymbol{\psi}\|^{2}=\sum_{j}\left|a_{j}(t)\right|^{2}$ at the $\operatorname{EP}(\epsilon \neq 0$ and $p=0)$ in a microring cavity under (a) a delta-function-like pulse with a period $T=4 \times 10^{5}$ and (b) a Gaussian noise input (red curves). These results were obtained from Eq. (11) with the $2 \times 2$ matrix of the microring cavity for $\gamma_{0} / \gamma_{n}=0.4$. For comparison, the intensity in a ring cavity without backscattering, i.e., a cavity at the DP $(\epsilon=$ $p=0)$, is also shown as the blue dotted curve for each case. In (b), $\boldsymbol{f}=\left(f_{1}, f_{2}\right)^{T}$ is treated as complex white Gaussian noise, $\left\langle f_{i}^{*}\left(t^{\prime}\right) f_{j}(t)\right\rangle=2 D \delta_{i j} \delta\left(t-t^{\prime}\right)$, where $D$ is the noise variance. (c) $\gamma_{0} / \gamma_{n}$ dependence of $I_{e p} / I_{d p}$. $I_{e p}$ indicates the time average of the response intensity dynamics at the EP, whereas $I_{d p}$ indicates that at the DP $(\epsilon=p=0)$. Gaussian noise is applied to the cavity.

medium [54, 55]:

$$
\begin{array}{r}
\frac{\partial E}{\partial t}=\frac{i}{2}\left[\frac{\partial^{2}}{\partial x^{2}}+\frac{n^{2}(x)}{n_{0}^{2}}\right] E+\xi \rho+E_{i n}, \\
\frac{\partial \rho}{\partial t}=-\left(\gamma_{\perp}+i \Delta_{a}\right) \rho+\gamma_{\perp} W E+F_{1}, \\
\frac{\partial W}{\partial t}=-\gamma_{\|}\left(W-W_{\infty}\right)-2 \gamma_{\|}\left(E \rho^{*}+E^{*} \rho\right)+F_{2},
\end{array}
$$

where space and time are made dimensionless by the scale transformations $n_{0} \omega_{s} x / c \rightarrow x$ and $\omega_{s} t \rightarrow t$, respectively. $\omega_{s}$ is a reference frequency close to the transition frequency $\omega_{a}$ of the two-level gain medium. In Eqs. (8)-(10), $E, \rho, W$, and all of the other parameters are also made dimensionless. Further, $n$ is the refractive index inside the cavity, $n_{0}$ is the spatially averaged refractive index, $\xi=2 \pi / n_{0}^{2}$ is a coupling constant, and $\Delta_{a}$ represents the gain center. (The relationship between $\Delta_{a}$ and the actual transition frequency $\omega_{a}$ is given by $\Delta_{a}=\omega_{a} / \omega_{s}-1$.) The two relaxation parameters, $\gamma_{\perp}$ and $\gamma_{\|}$, are the transverse and longitudinal relaxation rates, respectively. $W_{\infty}$ represents the pumping power, which is effectively used to reduce the cavity loss.

In Eq. (8), $E_{\text {in }}$ represents the input field, which is 
coupled to the intracavity field $E . F_{1}$ and $F_{2}$ represent spontaneous emission noise from the gain medium, and they are modeled as complex white Gaussian noise [56, 57]. The specific forms of $F_{1}$ and $F_{2}$ are similar to those reported in [57].

\section{B. Cavity model and parameters}

For verification, we choose a microring cavity, which is discussed in Sec. IIC. The cavity is modeled as a onedimensional ring waveguide with a length of $L$. A periodic boundary condition is imposed on the intracavity field as $E(x, t)=E(x+L, t)$, where $x$ denotes the coordinate along the ring waveguide. Tuning to an EP in the ring cavity is possible by modulating the complex-valued refractive index $n^{2}(x)$ inside the cavity [52]:

$$
n^{2}(x)=n_{0}^{2}\left[1+\epsilon \exp \left(2 i k_{0} x\right)+p \exp \left(-2 i k_{0} x\right)+2 i \beta\right]
$$

where $k_{0}=2 \pi m_{0} / L\left(m_{0}\right.$ is an integer $)$ represents the resonant wavenumber of the cavity for $\epsilon=p=0$, and $\beta$ represents the absorption loss rate. The refractive index modulation induces strong linear coupling between the nearly degenerate eigenmodes with the $+k_{0}(\mathrm{CCW})$ and $-k_{0}(\mathrm{CW})$ wave components. In particular, when $\epsilon \neq$ 0 and $p=0$, the two eigenmodes collapse to a single mode, which can be expressed as the CCW mode of the wavenumber $k_{0}$. Because the physical meanings of $\epsilon$ and $p$ are the same as those described in Sec. IIC, we use the same notation.

In this simulation, the following parameters are fixed: $L /(2 \pi)=10, n_{0}=3.0, k_{0}=1\left(m_{0}=10\right), \xi=2 \pi / n_{0}^{2}$, $\beta / \xi=10^{-3}, \Delta_{a}=0, \gamma_{\perp}=0.1$, and $\gamma_{\|}=10^{-3}$. For these parameters, the resonant frequency $\Delta_{0}$ corresponding to the wavenumber $k_{0}$ at the EP is set to be equal to the gain center $\Delta_{a}$, and the modes with $\Delta_{0}$ are selectively pumped. The effective decay rate, including the effect of the gain, is defined as $\gamma_{0}=\beta-\xi W_{\infty}$ 54 and is changed by $W_{\infty}$. In this paper, $W_{\infty}$ is kept below the threshold pumping power $W_{t h}=\beta / \xi$, and $\gamma_{0}$ is always positive.

\section{Response characteristics}

In this subsection, for simplicity, we omit the noise terms, $F_{1}$ and $F_{2}$, and we consider the case in which two input waves with a frequency of $\Delta$ are coupled to the cavity, as shown in Fig. 1, i.e., $E_{i n}=\kappa_{1} \exp \left(i k_{0} x-\right.$ $i \Delta t)+\kappa_{2} \exp \left(-i k_{0} x-i \Delta t\right)$, where $\kappa_{1}$ and $\kappa_{2}$ represent the CCW and CW wave components of the input waves in the cavity, respectively. To analyze the responses to the input waves, we measure the intensities of the CCW and CW wave components of the field $E$ as $I_{c c w}=\sum_{m>0}\left|a_{m}\right|^{2}$ and $I_{c w}=\sum_{m<0}\left|a_{m}\right|^{2}$, respectively, where $a_{m}=1 / L \int_{0}^{L} E(x, t) e^{-i k_{m} x} d x$, and $k_{m}=2 \pi m / L$ $(m \in \mathbb{Z})$, and calculate the time averages of $I_{c w}$ and $I_{c c w}$ after relaxation to a steady state.
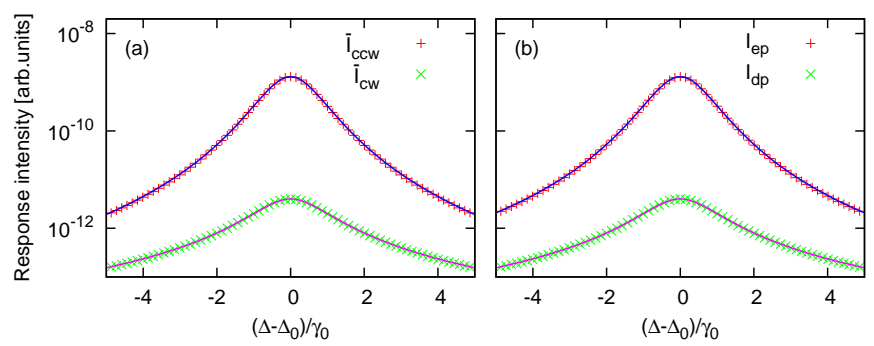

FIG. 3: (a) Time-averaged response intensities of the CCW and $\mathrm{CW}$ waves at the $\mathrm{EP}(\epsilon=1.8 \beta$ and $p=0), \bar{I}_{c c w}$ and $\bar{I}_{c w}$, under the $\mathrm{CW}$ wave input condition $\left(\kappa_{1}=0, \kappa_{2}=10^{-7} \beta\right)$. $\Delta_{0}$ is the degenerate resonant frequency at the EP. The theoretical curves of the $\mathrm{CCW}$ and $\mathrm{CW}$ wave intensities obtained from Eq. (6) are shown as the blue and pink solid curves, respectively. (b) Intensity $I_{e p}=\bar{I}_{c c w}+\bar{I}_{c w}$ at the EP under the same $\mathrm{CW}$ wave input condition. For comparison, the intensity $I_{d p}$ at the $\mathrm{DP}(\epsilon=p=0)$ is also shown. The theoretical curves obtained from Eq. (6) at the EP and DP are shown as the blue and pink solid curves, respectively. In (a) and (b), $W_{\infty}=0.95 \times 10^{-3}$, and $\gamma_{0} / \gamma_{s}=1 / 18$.
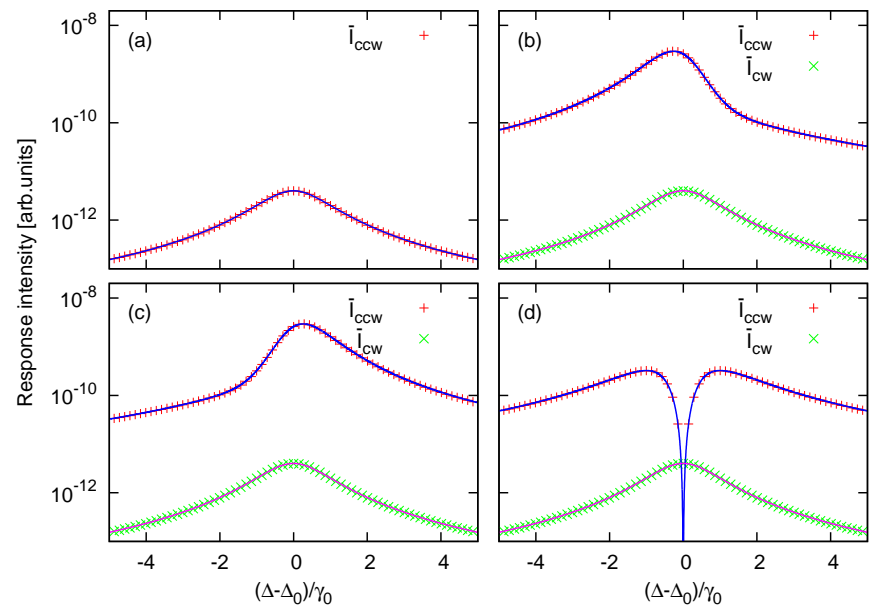

FIG. 4: (a) Time-averaged response intensity $\bar{I}_{c c w}$ under the CCW wave input condition $\left(\kappa_{1}=10^{-7} \beta\right.$ and $\left.\kappa_{2}=0\right)$. (b)(d) Time-averaged response intensities $\bar{I}_{c c w}$ and $\bar{I}_{c w}$ under a bidirectional wave input condition with (b) $\kappa_{1}=\epsilon /\left(2 \gamma_{0}\right) \kappa_{2}$, (c) $\kappa_{1}=-\epsilon /\left(2 \gamma_{0}\right) \kappa_{2}$, and (d) $\kappa_{1}=-i \epsilon /\left(2 \gamma_{0}\right) \kappa_{2}$, where $\kappa_{2}=10^{-7} \beta$. In (a)-(d), $\epsilon=1.8 \beta, p=0, W_{\infty}=0.95 \times 10^{-3}$, and $\gamma_{0} / \gamma_{s}=1 / 18$. The theoretical curves of the CCW and CW wave intensities obtained from Eq. (6) for each input condition are shown as the blue and pink solid curves, respectively.

Here, we consider the responses at the $\operatorname{EP}(\epsilon \neq 0$ and $p=0$ ) under the $\mathrm{CW}$ wave input condition, i.e., $\kappa_{1}=0$ and $\kappa_{2} \neq 0$. In Fig. [3(a), the time-averaged intensities $\bar{I}_{c c w}$ and $\bar{I}_{c w}$ under the input condition are plotted as a function of the frequency $\Delta . \epsilon$ and $W_{\infty}$ are set so that $\gamma_{0}$ is smaller than the critical value $\gamma_{s}(=|\epsilon| / 2)$, which is provided in Sec. II C. For a relatively low input intensity, i.e., $\kappa_{2}=10^{-7} \beta$, the numerical results correspond well to the theoretical results obtained from Eq. (6); 


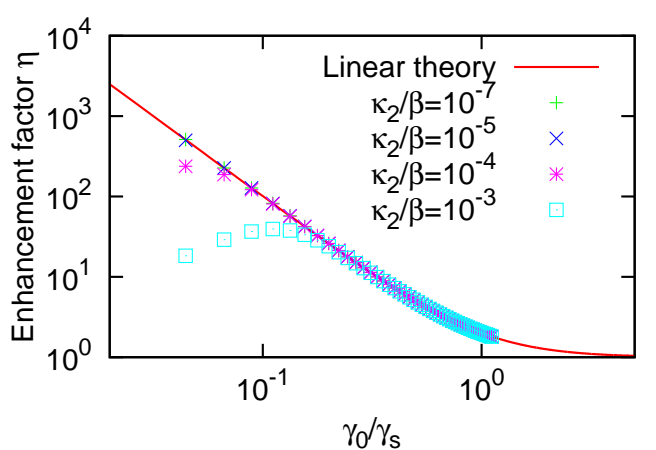

FIG. 5: Enhancement factor $\eta$ versus $\gamma_{0} / \gamma_{s} . \eta$ is measured under the CW wave input condition $\left(\kappa_{1}=0\right)$ at the resonance $\Delta=\Delta_{0}$. The theoretical curve is given by $\eta=\gamma_{s}^{2} / \gamma_{0}^{2}+1$.

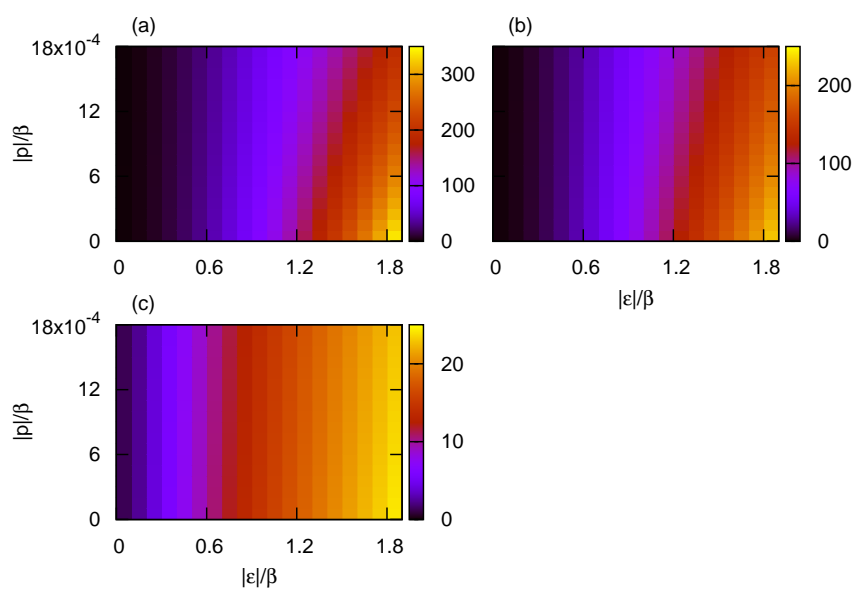

FIG. 6: Enhancement factor $\eta$ plotted in the $\epsilon-p$ plane. (a) $\kappa_{2}=10^{-7} \beta$, (b) $\kappa_{2}=10^{-4} \beta$, and (c) $\kappa_{3}=10^{-3} \beta$. In (a)-(c), $W_{\infty}=0.95 \times 10^{-3}$, and $\arg (\epsilon / p)=0$.

the frequency response intensities of the $\mathrm{CCW}$ and $\mathrm{CW}$ waves have a squared Lorentzian shape and conventional Lorentzian shape, respectively. We can see that the CCW wave intensity at the resonance $\left(\Delta=\Delta_{0}\right)$ is two orders of magnitude greater than the $\mathrm{CW}$ wave intensity [see Fig. [3(a)].

According to Eq. (6), the response characteristics at an EP can be controlled by varying the input waves. For example, under the CCW wave input condition $\left(\kappa_{1} \neq 0\right.$ and $\kappa_{2}=0$ ), the CCW wave intensity is not enhanced, as shown in Fig. 4(a), and the frequency response intensity has a Lorentzian shape. On the other hand, when two input waves with well-controlled phases and amplitudes are simultaneously coupled to the cavity, the interference of the two input waves leads to an asymmetric response curve for $\kappa_{1}= \pm \epsilon /\left(2 \gamma_{0}\right) \kappa_{2}$ [Figs. 4(b) and 4(c)] or suppression of the response intensity at the resonance $\left(\Delta=\Delta_{0}\right)$ for $\kappa_{1}=-i \epsilon /\left(2 \gamma_{0}\right) \kappa_{2}$ [Fig. [4(d)]. This suggests the possibility of novel mode switching; however, its further study is beyond the scope of this paper.
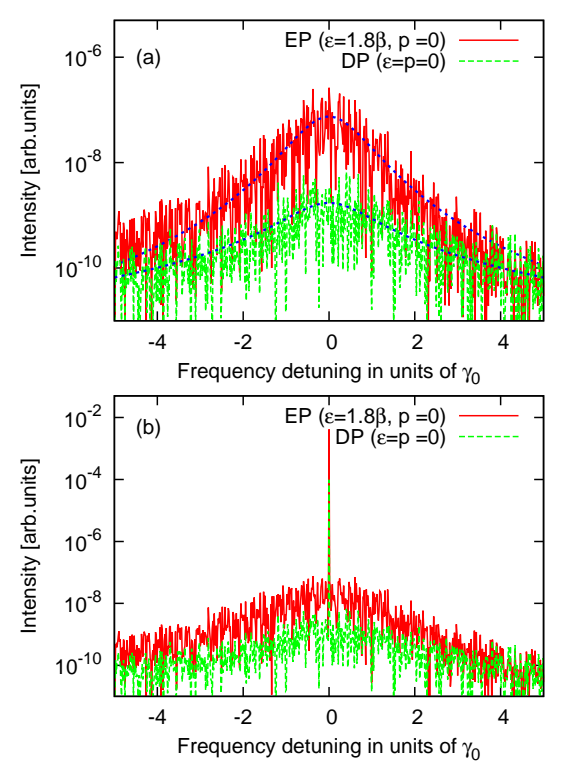

FIG. 7: (a) Each emission spectrum at the EP $(\epsilon=1.8 \beta$ and $p=0)$ and DP $(\epsilon=p=0)$. The spectrum at the EP is fitted by a squared Lorentzian curve, whereas the spectrum at the DP is fitted by a Lorentzian curve. The fitting curves are shown as the blue dotted curves. (b) Emission spectra when an input wave with a frequency of $\Delta_{0}$ is coupled to the cavity in the CW direction. In (a) and (b), $\kappa=10^{-3} \beta$, $W_{\infty}=0.9 \times 10^{-3}$, and $\gamma_{0} / \gamma_{n}=2 / 9$.

\section{Response enhancement and its limitations}

In what follows, we consider only the $\mathrm{CW}$ wave input condition $\left(\kappa_{1}=0\right.$ and $\left.\kappa_{2} \neq 0\right)$ and compare the response intensity at the EP to that in a ring cavity with the same radius, the same loss rate $\gamma_{0}$, and the same coupling strength $\kappa_{2}$ but without non-Hermitian backscattering, i.e., the response intensity at the $\mathrm{DP}(\epsilon=p=0)$.

Figure 3(b) shows an example that compares the frequency response intensity at the $\mathrm{EP}, I_{e p}$, and that at the $\mathrm{DP}, I_{d p}$, which are calculated as $\bar{I}_{c c w}+\bar{I}_{c w}$. To quantitatively evaluate the enhancement, the enhancement factor $\eta$ was defined as the ratio of each peak intensity at the resonance $\left(\Delta=\Delta_{0}\right)$, i.e., $\eta=I_{e p} / I_{d p}$. For $\kappa_{2}=10^{-7} \beta$ and $\gamma_{0} / \gamma_{s}=1 / 18$, we obtain $\eta \approx 325$.

The parametric dependence of $\eta$ is summarized in Figs. 5 and 6. For a low input intensity, i.e., $\kappa_{2}=10^{-7} \beta_{0}, \eta$ is inversely proportional to $\gamma_{0}^{2}$ when $\gamma_{0} / \gamma_{s}<1$ (Fig. [5), and it is proportional to $|\epsilon|^{2}$ [Fig. 6(a)], as predicted by the linear theory. However, for a relatively large input intensity, i.e., $\kappa_{2}>10^{-4} \beta$, the enhancement is reduced. This is the result of the nonlinear gain saturation, because in our case, gain saturation has a significant effect on the intensity amplification when $\gamma_{0} \ll \sqrt{\left|\epsilon \kappa_{2}\right|}$, according to the nonlinear steady-state analysis of Eqs. (8)-(10). The gain saturation may also change the positions of the EPs in the parametric spaces. Consequently, the saturation effect limits the enhancement in the response intensity. 
However, we note that it also makes the enhancement less sensitive to the deviation from the EPs (i.e., $|p|>0$ ) [see Figs. 6(b) and (c)].

\section{E. Effects of spontaneous emission noise}

As demonstrated in the previous subsections, the reduction in the decay rate $\gamma_{0}$ by the gain is indispensable for enhancing the response intensity. However, the presence of the gain inevitably results in spontaneous emission noise; therefore, the response to the input waves as well as the spontaneous emission may both be enhanced if the condition in Eq. (7) is satisfied. An example of the enhanced spontaneous emission spectrum for $\gamma_{0}<\gamma_{n}$ at the EP is shown in Fig. 7(a). The spectrum was calculated by taking into account the noise terms $F_{1}$ and $F_{2}$ in Eqs. (8) - (10) with no input waves, i.e., $\kappa_{1}=\kappa_{2}=0$. The figure also shows the fitting curves, which were obtained by comparing the integrals of the numerical spectra and the theoretical curves (see Appendix B for the details). One can see that the emission spectrum at the EP can be well-fitted by a squared Lorentzian curve, and the amplitude is greater than that of the spectrum measured at the DP. The squared Lorentzian shape is general at a second-order EP in any non-Hermitian photonic system with gain, and it can be experimentally observed.

Figure 7(b) shows the spectra under the CW wave input condition at the resonance $\Delta=\Delta_{0}$. One can clearly see that the response intensity to the input wave at the EP can be more than an order of magnitude greater than that at the DP, although the spontaneous emission is also enhanced. The signal-to-noise ratio is not degraded in this case. These results suggest the experimental realization of the enhancement in the response to input signals by using EPs.

\section{SUMMARY AND DISCUSSION}

The response characteristics of non-Hermitian optical cavities near EPs were analytically studied on the basis of Eq. (4). Although the $K$-factor (PF) diverges at these points, the actual response amplitude is limited to a finite value. Importantly, optical cavities operating at an EP can exhibit a non-Lorentzian frequency response due to the interference between the first and second poles in the resolvent, which mainly depends on the decay rate $\gamma_{0}$, the input field $\boldsymbol{f}$, and the non-Hermitian system parameters $c_{i j}$ [see Eq. (44)]. This is a unique property of systems operating near an EP. When a system and the input channel are appropriately designed and the decay rate $\gamma_{0}$ is reduced by gain, one can observe a significant enhancement in the response near the coalescing resonance at the EP compared to normal systems with the same loss and same coupling parameters for the input waves. The results of the linear theory were numerically verified in a microring cavity with non-Hermitian backscattering by using a dynamical model taking into account the effects of the gain and spontaneous emission. With the aid of the gain, the intracavity intensity can respond strongly to an input field as well as optical noise. Although the gain saturation occurring under a strong input field leads to a decrease in the response intensity, the intensity is still much higher than that of a standard optical cavity that has the same input wave coupling strength to each eigenmode but does not operate at the EP. Even when the spontaneous emission is enhanced, the signal-to-noise ratio does not decrease because of the excess response to input signals, as far as a gain saturation effect is not dominant. These results suggest the possibility of experimental observation of the enhanced response behavior by using EPs. For example, an enhancement in a microring cavity operating at an EP could be observed in an add-drop configuration with input and output waveguides if asymmetric backscattering is so strong that the condition $\gamma_{0}<\gamma_{s}$ is satisfied.

A further enhancement is possible if a system can operate at a higher-order EP because the resolvent or Green's function has higher-order poles [48, 58].

The response theory presented in this work is applicable to a variety of photonic systems, including optical microcavities, parity-time symmetric systems, optomechanical resonators, and plasmonic systems that can operate at an EP. The response characteristics at an EP shown in this work, e.g., the enhancement in or suppression of the response amplitude, will be useful for controlling the excitation of eigenmodes, the output from resonator sensors at EPs 37 40, extraordinary optical transmission [59], or the light-matter interactions inside microcavities.

\section{Acknowledgments}

I would like to thank Professor T. Niyama and Dr. J.W. Ryu for reading the manuscript and offering valuable comments. This work was supported by JSPS KAKENHI, Grant No. 16K04974.

\section{Appendix A: Derivation of Eq. (4)}

The resolvent $\hat{M}_{E P}$ at an EP is derived by assuming that $\hat{M}$ can be described by a $2 \times 2$ matrix involving a pair of nearly degenerate modes, $j=1$ and $j=2$. Although expressions similar to $\hat{M}_{E P}$ have been reported in Ref. [45, 46, 58], the $2 \times 2$ matrix form derived in this work allows for a simple insight into the relationship between the response functions and the matrix $\hat{H}$.

Here, let $\Omega_{j}$ and $\boldsymbol{u}_{j}$ be an eigenvalue and the right eigenvector of mode $j$, respectively. The resolvent $\hat{M}$ is rewritten using an eigenvector matrix $\hat{P}=\left(\boldsymbol{u}_{1}, \boldsymbol{u}_{2}\right)$ as 
follows:

$$
\begin{gathered}
\hat{M}(\omega)=(\omega \hat{I}-\hat{H})^{-1}=\hat{P}(\omega \hat{I}-\hat{\Lambda})^{-1} \hat{P}^{-1} \\
=\left(\begin{array}{cc}
R_{1}+\frac{R_{1}-R_{2}}{\operatorname{det} \hat{P}} u_{12} u_{21} & -\frac{R_{1}-R_{2}}{\operatorname{det} \hat{P}} u_{11} u_{12} \\
\frac{R_{1}-R_{2}}{\operatorname{det} \hat{P}} u_{21} u_{22} & R_{2}-\frac{R_{1}-R_{2}}{\operatorname{det} \hat{P}} u_{12} u_{21}
\end{array}\right)
\end{gathered}
$$

where $\hat{\Lambda}=\operatorname{diag}\left(\Omega_{1}, \Omega_{2}\right), R_{j}=\left(\omega-\Omega_{j}\right)^{-1}$, and $\boldsymbol{u}_{j}=$ $\left(u_{1 j}, u_{2 j}\right)^{T}$. Note that $\operatorname{det} \hat{P}$ and $R_{1}-R_{2}$ both become zero at an EP, where the two eigenvalues coincide, and their eigenvectors completely overlap each other. The convergence of $\left(R_{1}-R_{2}\right) / \operatorname{det} \hat{P}$ can be analyzed by applying perturbation theory near an EP to the above equation [45]. Suppose that $\hat{H}_{0}, \Omega_{0}$, and $\boldsymbol{u}_{0}=\left(u_{10}, u_{20}\right)^{T}$ are the Hamiltonian at an EP, the coalesced eigenvalue, and the corresponding eigenvector, respectively. According to [43, 44], $\Omega_{j}$ and $\boldsymbol{u}_{j}$ can be expressed as $\Omega_{0} \pm \Delta \Omega$ and $\boldsymbol{u}_{j}=\boldsymbol{u}_{0} \pm \Delta \Omega \boldsymbol{u}_{J}+O\left(|\Delta \Omega|^{2}\right)$, respectively, near an EP. $\Delta \Omega$ is the deviation from $\Omega_{0}$, and $\boldsymbol{u}_{J}=\left(u_{1 J}, u_{2 J}\right)^{T}$ is an associated vector, which satisfies a generalized eigenvalue equation, $\left(\hat{H}_{0}-\Omega_{0} \hat{I}\right) \boldsymbol{u}_{J}=\boldsymbol{u}_{0}$. By using $\boldsymbol{u}_{0}$ and the associated vector $\boldsymbol{u}_{J}$, $\operatorname{det} \hat{P}$ is obtained as $-2 \Delta \Omega J+O\left(|\Delta \Omega|^{2}\right)$, where $J$ is the determinant of the matrix $\hat{P}_{J}=\left(\boldsymbol{u}_{0}, \boldsymbol{u}_{J}\right)$. We omit the second order of $|\Delta \Omega|^{2}$ near an EP and obtain

$$
\hat{M}(\omega) \approx\left(\begin{array}{cc}
R_{a}+c_{11} R_{d} & c_{12} R_{d} \\
c_{21} R_{d} & R_{a}+c_{22} R_{d}
\end{array}\right),
$$

where $R_{a}=\left(R_{1}+R_{2}\right) / 2$ and

$$
\begin{aligned}
R_{d} & =\frac{1}{2 \Delta \Omega}\left(\frac{1}{\omega-\Omega_{1}}-\frac{1}{\omega-\Omega_{2}}\right) \\
& =\frac{1}{\left(\omega-\Omega_{0}\right)^{2}-\Delta \Omega^{2}} .
\end{aligned}
$$

When $\Delta \Omega \rightarrow 0, R_{a} \rightarrow R_{0}$, and $R_{d} \rightarrow R_{0}^{2}$. In the above, $c_{11}=-u_{10} u_{20} / J, c_{12}=u_{10}^{2} / J, c_{21}=-u_{20}^{2} / J$, and $c_{22}=-c_{11}$. These coefficients correspond to the matrix components of $\left(\hat{H}_{0}-\Omega_{0} \hat{I}\right)$. This can be easily confirmed by calculating $\left(\hat{H}_{0}-\Omega_{0} \hat{I}\right)=\hat{P}_{J}\left(\hat{T}_{0}-\Omega_{0} \hat{I}\right) \hat{P}_{J}^{-1}$, where

$$
\hat{T}_{0}=\left(\begin{array}{cc}
\Omega_{0} & 1 \\
0 & \Omega_{0}
\end{array}\right) .
$$

\section{Appendix B: Fitting of the spontaneous emission spectra}

Because the emission spectra shown in Fig. 7(a) fluctuate, the integrals of the spectra are compared to those of the theoretical spectra. This fitting method is similar

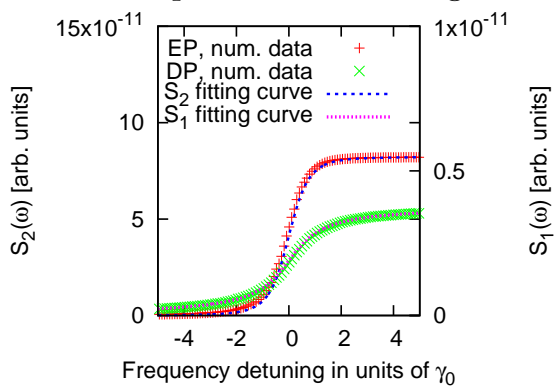

FIG. 8: Integrals of the noise spectra shown in Fig. 7(a). The integrals of the spectra at the EP and DP are fitted by $S_{1}$ and $S_{2}$, respectively.

to that used in Ref. [57]. According to the linear theory presented in Sec. III, the emission spectrum at the EP is represented by a squared Lorentzian curve when $\gamma_{0} \ll \gamma_{n}$, whereas the spectrum at the $\operatorname{DP}(\epsilon=p=0)$ is a Lorentzian curve. The integral of a Lorentzian curve, $L(\omega)=A_{1} /\left[\gamma_{0}^{2}+\left(\omega-\Delta_{0}\right)^{2}\right]$, is

$$
S_{1}(\omega)=\int_{-\infty}^{\omega} L(\omega) d \omega=\frac{A_{1}}{\gamma_{0}}\left[\theta(\omega)+\frac{\pi}{2}\right],
$$

where $A_{1}$ is a fitting parameter, and $\theta(\omega)=\tan ^{-1}[(\omega-$ $\left.\left.\Delta_{0}\right) / \gamma_{0}\right]$. The integral of a squared Lorentzian curve, $L_{2}(\omega)=A_{2}|\epsilon|^{2} /\left(4\left[\gamma_{0}^{2}+\left(\omega-\Delta_{0}\right)^{2}\right]^{2}\right)$, is

$$
\begin{aligned}
S_{2}(\omega) & =\int_{-\infty}^{\omega} L_{2}(\omega) d \omega \\
& =\frac{A_{2}|\epsilon|^{2}}{8 \gamma_{0}^{3}}\left[\theta(\omega)+\frac{1}{2} \sin 2 \theta(\omega)+\frac{\pi}{2}\right],
\end{aligned}
$$

where $A_{2}$ is a fitting parameter. The fitting results are shown in Fig. 8 .
[1] J. Okolowicz, M. Ploszajczak, and I. Rotter, Phys. Rep. 374, 271 (2003).

[2] L. N. Trefethen and M. Embree, Spectra and Pseudospectra: The Behavior of Nonnormal Matrices and Operators (Princeton University Press, Princeton, NJ, 2005).

[3] L. N. Trefethen, A. E. Trefethen, S. C. Reddy and T. A.
Driscoll, Science 261, 578-584 (1993).

[4] Brian F. Farrell and Petros J. Ioannou, Phys. Rev. Lett. 721188 (1994).

[5] G. Szirmai, D. Nagy, and P. Domokos, Phys. Rev. Lett. 102, 080401 (2009).

[6] F. Papoff, G. D'Alessandro, and G.-L. Oppo, Phys. Rev. 
Lett. 100, 123905 (2008).

[7] S. Longhi and P. Laporta, Phys. Rev. E 61, R989(R) (2000).

[8] G. D'Alessandro and F. Papoff, Phys. Rev. A 80, 023804 (2009).

[9] Tommaso Biancalani, Farshid Jafarpour, and Nigel Goldenfeld, Phys. Rev. Lett. 118, 018101 (2017).

[10] K. Petermann, IEEE J. Quantum Electron. QE-15, 566 (1979).

[11] H. A. Haus and S. Kawakami, IEEE J. Qunatum Electron. 21, 63 (1985).

[12] A. E. Siegman, Phys. Rev. A 39, 1253 (1989); 39, 1264 (1989).

[13] A. E. Siegman, Appl. Phys. B 60, 247 (1995).

[14] M. P. van Exter, N. J. van Druten, A. M. van der Lee, S. M. Dutra, G. Nienhuis, and J. P. Woerdman, Phys. Rev. A 63, 043801 (2001).

[15] Yuh-Jen Cheng, Phys. Rev. Lett. 97, 093601 (2006).

[16] S.-Y. Lee, J.-W. Ryu, J.-B. Shim, S.-B. Lee, S. W. Kim, and K. An, Phys. Rev. A 78, 015805 (2008).

[17] Yuh-Jen Cheng, C. G. Fanning, and A. E. Siegman, Phys. Rev. Lett. 77, 627 (1996).

[18] M. A. van Eijkelenborg, A. M. Lindberg, M. S. Thijssen, and J. P. Woerdman, Phys. Rev. Lett. 77, 4314 (1996).

[19] Gang Yao, Y. C. Chen, C. M. Harding, S. M. Sherrick, R. J. Dalby, R. G. Waters, and C. Largent, Opt. Lett. 17, 1207-1209 (1992).

[20] A. M. van der Lee, N. J. van Druten, A. L. Mieremet, M. A. van Eijkelenborg, A. M. Lindberg, M. P. van Exter, and J. P. Woerdman, Phys. Rev. Lett. 79, 4357 (1997).

[21] A. M. van der Lee, A. L. Mieremet, M. P. van Exter, N. J. van Druten, and J. P. Woerdman, Phys. Rev. A 61, 033812 (2000).

[22] T. Kato, Perturbation theory for Linear Operators (Springer, New York, 1966).

[23] M. V. Berry, Czech. J. Phys. 54, 1039-1047 (2004).

[24] W. D. Heiss, J. Phys. A: Math. Theor. 45, 444016 (2012).

[25] H. Cao and J. Wiersig, Rev. Mod. Phys. 87, 61-111 (2015).

[26] S.-B. Lee, J. Yang, S. Moon, S.-Y. Lee, J.-B. Shim, S. W. Kim, J.-H. Lee, and K. An, Phys. Rev. Lett. 103, 134101 (2009).

[27] B. Zhen, C. W. Hsu, Y. Igarashi, L. Lu, I. Kaminer, A. Pick, S.-L. Chua, J. D. Joannopoulos, and M. Soljačić, Nature 525, 354-358 (2015).

[28] K.-H. Kim, M.-S. Hwang, H.-R. Kim, J.-H. Choi, Y.-S. No, and H.-G. Park, Nat. Commun. 713893 (2016).

[29] J. Doppler, A. A. Mailybaev, J. Böhm, U. Kuhl, A. Girschik, F. Libisch, T. J. Milburn, P. Rabl, N. Moiseyev and S. Rotter, Nature 537, 76-79 (2016).

[30] M. Liertzer, Li Ge, A. Cerjan, A. D. Stone, H. E. Türeci, and S. Rotter, Phys. Rev. Lett. 108, 173901 (2012).

[31] B. Peng, Ş. K. Özdemir, S. Rotter, H. Yilmaz, M. Liertzer, F. Monifi, C. M. Bender, F. Nori, and L. Yang, Science 346, 328 (2015).

[32] B. Peng, Ş. K. Özdemir, F. Lei, F. Monifi, M. Gianfreda, G. L. Long, S. Fan, F. Nori, C. M. Bender, and L. Yang, Nat. Phys. 10, 394 (2014).

[33] L. Chang, X. Jiang1, S. Hua1, C. Yang, J. Wen, L. Jiang, G. Li, G. Wang, and M. Xiao, Nat. Photon. 8, 524 (2014).

[34] Z. Lin, H. Ramezani, T. Eichelkraut, T. Kottos, H. Cao, and D. N. Christodoulides, Phys. Rev. Lett. 106, 213901 (2011).

[35] L. Feng, Y.-L. Xu, W. S. Fegadolli, M.-H. Lu, J. E. B. Oliveira, V. R. Almeida, Y.-F. Chen, and A. Scherer, Nat. Mater. 12(2), 108 (2013).

[36] J. Wiersig, Phys. Rev. Lett 112, 203901 (2014); J. Wiersig, Phys. Rev. A 93, 033809 (2016).

[37] W. Chen, Ş. K. Özdemir, G. Zhao, J. Wiersig, and L. Yang, Nature 548, 192 (2017).

[38] H. Hodaei, A. U. Hassan, S. Wittek, H. Garcia-Gracia, R. El-Ganainy, D. N. Christodoulides, and M. Khajavikhan, Nature, 548(7666) 187 (2017).

[39] J. Ren, H. Hodaei, G. Harari, A. U. Hassan, W. Chow, M. Soltani, D. N. Christodoulides, and M. Khajavikhan, Opt. Lett. 42, 1556 (2017).

[40] S. Sunada, Phys. Rev. A 96, 033842 (2017).

[41] J. Demmel, Applied Numerical Linear Algebra (SIAM, Philadelphia, 1997).

[42] In other words, $K_{j}$ is 1 only for normal systems, where the set of the eigenvectors forms an orthogonal basis, and $\boldsymbol{u}_{j}$ can be parallel to $\boldsymbol{v}_{j}$, e.g., see [41].

[43] A. P. Seyranian and A. A. Mailybaev, Multiparameter Stability Theory with Mechanical Applications (Singapore: World Scientific, 2003).

[44] A. P. Seyranian, O. N. Kirillov and A. A. Mailybaev, J. Phys. A: Math. Gen. 38, 1723 (2005).

[45] A. Pick, B. Zhen, O. D. Miller, C. W. Hsu, F. Hernandez, A. W. Rodriguez, M. Soljačić, and S. G. Johnson, Opt. Express 25(11), pp. 12325-12348 (2017).

[46] W. D. Heiss and G. Wunner, Eur. Phys. J. D 68, 284 (2014).

[47] W. Suh, Z. Wang, and S. Fan, IEEE J. Quantum Electron. 40, 1511 (2004).

[48] Z. Lin, A. Pick, M. Lončar, and A. W. Rodriguez, Phys. Rev. Lett. 117, 107402 (2016).

[49] G. Yoo, H.-S. Sim, and H. Schomerus, Phys. Rev. A 84, 063833 (2011).

[50] B. Peng, S. K. Özdemir, M. Liertzer, W. Chen, J. Kramer, H. Yilmaz, J. Wiersig, S. Rotter, and L. Yang, PNAS 113, 6845 (2016).

[51] J. Wiersig, A. Eberspacher, J.-B. Shim, J.-W. Ryu, S. Shinohara, M. Hentschel, and H. Schomerus Phys. Rev. A 84, 023845 (2011).

[52] F.-J. Shu, C.-L. Zou, X.-B. Zou, and L. Yang, Phys. Rev. A 94, 013848 (2016).

[53] B. F. Farrell and P. J. Ioannou, J. Atmos. Sci. 53, 2025 (1996).

[54] T. Harayama, S. Sunada, and K. S. Ikeda, Phys. Rev. A 72, 013803 (2005).

[55] S. Sunada, T. Fukushima, S. Shinohara, T. Harayama, and M. Adachi, Phys. Rev. A 88, 013802 (2013).

[56] P. D. Drummond and M. G. Raymer, Phys. Rev. A 44, 2072 (1991).

[57] A. Cerjan, A. Pick, Y. D. Chong, S. G. Johnson, and A. D. Stone, Opt. Express 23, 28316 (2015).

[58] W. D. Heiss, Int. J. Theor. Phys. 543954 (2015).

[59] H-X. Cui, X.-W. Cao, M. Kang, T.-F. Li, M. Yang, T.-J. Guo, Q.-H. Guo, and J. Chen, Opt. Express 21, 13368 (2013). 